\title{
Optimized Adjustment of Single Action-potentials to Case-specific Atrial Physiology: Towards Clinical Implementation
}

\author{
Yvonne Richter ${ }^{1}$, Pedro G. Lind ${ }^{1}$, Gunnar Seemann ${ }^{2,3}$, Claudia Lenk ${ }^{4}$, Philipp Maass ${ }^{1}$ \\ ${ }^{1}$ Fachbereich Physik, Universität Osnabrück, Germany \\ ${ }^{2}$ Institute for Experimental Cardiovascular Medicine, University Heart Center Freiburg, Germany \\ ${ }^{3}$ Faculty of Medicine, University of Freiburg, Germany \\ ${ }^{4}$ Institut für Mikro- und Nanoelektronische Systeme, Technische Universität Ilmenau, Germany
}

\begin{abstract}
We present a method for modelling case-specific action potentials (APs) based on a simple model for electric wave propagation combined with an optimization procedure that searches the best combination of three model parameter values for a given action potential. The method is applied to a set of 70 APs, showing deviations around 10\%. This method can be useful for prognosing possible pathological scenarios of a specific patient, based on data from electrocardiagrams.
\end{abstract}

\section{Introduction}

For modeling the propagation of action potentials in the human atria, various models have been developed, which take into account the influence of the numerous ionic currents flowing through the cell membrane. Aiming at a simplified description, the Bueno-Orovio-Cherry-Fenton (BOCF) model [1] for electric wave propagation in the ventricle has been adapted to atrial physiology [2].

Recently, we studied this adapted BOCF model with respect to its capability to accurately generate spatiotemporal excitation patterns found in anatomical and spiral wave reentry [3]. We found that characteristic features of the reentrant excitation patterns seen in the more detailed model proposed by Courtemanche, Ramirez and Nattel (CRN) model [4] are well captured by the BOCF model. In particular, we showed that the size and conduction velocity of the spiral waves produced with both models differ by a constant difference smaller than $10 \%$. Moreover, we also compared APs generated with the BOCF model with APs produced with the CRN model [5] and discussed strengths of the BOCF model, namely its lower computational costs for extensive simulations and its reduced number of differential equations.

A natural step forward is to use these strengths for parameter adjustment to patient-specific electrophysiological
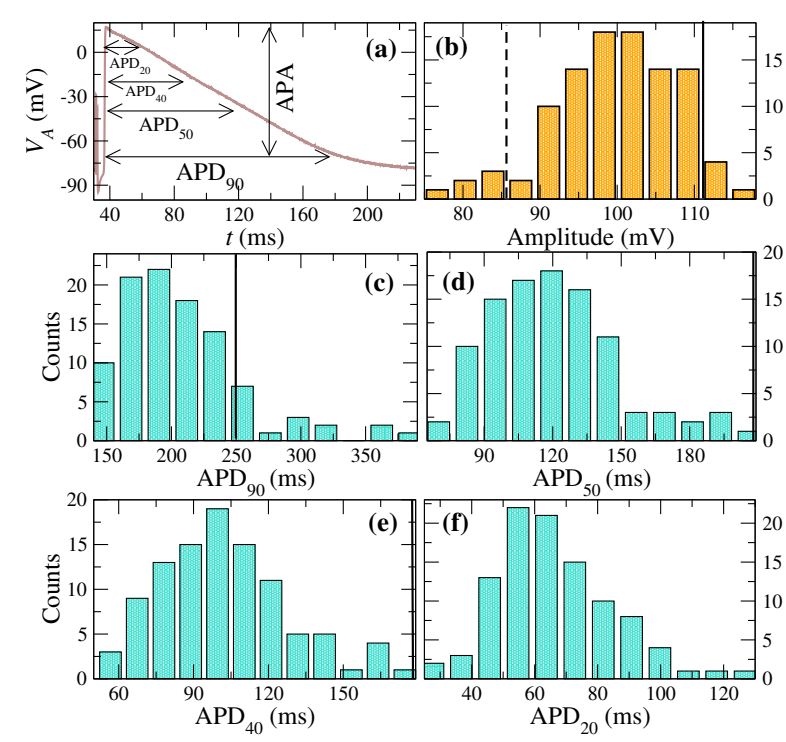

Figure 1. (a) Illustration of one AP and the five properties used to characterize it. (b) Distribution of the amplitudes of the 103 APs (see text) together with (c)-(f) the distribution of the AP durations at $90 \%, 50 \%, 40 \%$ and $20 \%$ of the APA, respectively. Vertical solid and dashed lines indicate respectively maximum and minimum limiting values of the admissible range that can be properly modelled with the BOCF model (see Fig. 2).

conditions. Here, we introduce such method to model specific action potentials with a pre-given amplitude, duration and shape by only adjusting a few parameters.

The specific data to be analyzed comprehends a set of 103 APs collected at the University of Dresden in the group of Prof. U. Ravens. Figure 1(a) shows one AP that we characterize by its action potential amplitude (APA) and AP durations (APDs) at four amplitude levels, namely at $90 \%, 50 \%, 40 \%$ and $20 \%$ of the APA. The distribution of each of these five AP properties for the full set of APs 
is given in Figs. 1(b)-(f).

\section{The model}

The BOCF model has four state variables. One is the transmembrane voltage $V$, that is rescaled to a normalized voltage $u$, such that $V=V_{R}(1+\alpha u)$, where $V_{R}=-84.1 \mathrm{mV}$ is the resting voltage, and $\alpha=1.02$ [2]. The three other variables $v, w$ and $s$ describe the gating of (effective) net currents through the cell membrane. These four state variables evolve according to a system of reaction-diffusion equations,

$$
\begin{aligned}
\partial_{t} u= & D \Delta u+J_{\mathrm{fi}}(u, v)+J_{\mathrm{si}}(u, w, s) \\
& +J_{\mathrm{so}}(u)+J_{\mathrm{stim}}, \\
\partial_{t}(v, w, s)= & (E(u, v), F(u, w), G(u, s)),
\end{aligned}
$$

where $J_{\mathrm{fi}}(u, v)$ represents a fast inward sodium-like current, $J_{\mathrm{si}}(u, w, s)$ a slow inward calcium-like current, and $J_{\text {so }}(u)$ a slow outward potassium-like current; $J_{\text {stim }}$ is an external stimulus current.

Defining by $H_{a}(x)$ the Heaviside jump function at $x=$ $a\left(H_{a}(x)=1\right.$ for $x \geq a$ and zero otherwise) and $H^{a}(x)=$ $1-H_{a}(x)$, the currents and the functions $E, F$ and $G$ in Eq. (1b) are

$$
\begin{aligned}
& J_{\mathrm{fi}}=\frac{v}{\tau_{\mathrm{fi}}}\left(u-\theta_{v}\right)\left(u_{u}-u\right) H_{\theta_{v}}(u), \\
& J_{\mathrm{si}}=\frac{w s}{\tau_{\mathrm{si}}} H_{\theta_{w}}(u), \\
& J_{\mathrm{so}}=-\frac{u}{T_{o}(u)} H^{\theta_{w}}(u) \\
& -\frac{H_{\theta_{w}}(u)}{\tau_{\mathrm{so} 1}+\left(\tau_{\mathrm{so} 2}-\tau_{\mathrm{so} 1}\right) Q_{\mathrm{so}}(u)}, \\
& J_{\text {stim }}=j_{\text {stim }}\left(H_{0}\left(t^{*}\right)-H_{0}\left(t^{*}+T\right)\right) \text {, } \\
& E(u, v)=-\frac{v-H^{\theta_{v}^{-}}(u)}{T_{v}^{-}(u)} H^{\theta_{v}}(u) \\
& -\frac{v}{\tau_{v}^{+}} H_{\theta_{v}}(u) \text {, } \\
& F(u, w)=-\frac{w-w_{\infty}}{\tau_{w 1}^{-}+\left(\tau_{w 2}^{-}-\tau_{w 1}^{-}\right) Q_{w}^{-}(u)} H^{\theta_{w}}(u) \\
& -\frac{w}{\tau_{w}^{+}} H_{\theta_{w}}(u), \\
& G(u, s)=\frac{1}{T_{\theta_{w}}(u)}\left(Q_{s}(u)-s\right),
\end{aligned}
$$

where

$$
T_{x}(u)=\tau_{x 1} H^{\theta_{x}}(u)+\tau_{x 2} H_{\theta_{x}}(u),
$$

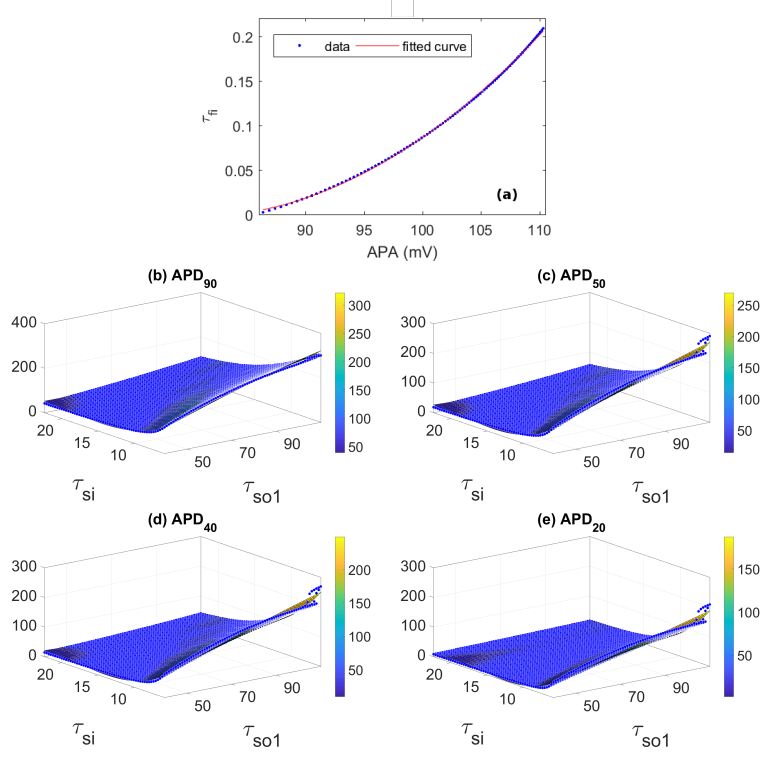

Figure 2. (a) Time-scale $\tau_{\text {fi }}$ as a function of the APA for $\tau_{\mathrm{si}}=10.7 \mathrm{~ms}$ and $\tau_{\mathrm{so} 1}=73.7 \mathrm{~ms}$. (b)-(e) Dependence of the four $\mathrm{APD}_{n}$ on $\tau_{\mathrm{si}}$ and $\tau_{\mathrm{so} 1}$ for fixed $\tau_{\mathrm{fi}}=0.0835 \mathrm{~ms}$. The meshes of points (black bullets) indicate the simulation results, and the surfaces refer to the fits of the meshes. All quantities are given in ms.

$$
\begin{aligned}
Q_{x}(u) & =\frac{1}{2}\left(1+\tanh \left[k_{x}\left(u-u_{x}\right)\right]\right), \\
w_{\infty} & =\left(1-\frac{u}{\tau_{w \infty}}\right) H^{\theta_{o}}(u)+w_{\infty}^{*} H_{\theta_{o}}(u) .(3 \mathrm{c})
\end{aligned}
$$

Here, $u_{u}=1.0089$ and $u_{\text {so }}=0.592093$ are reference values, $\theta_{v}=0.3$ and $\theta_{w}=0.18171$ are threshold values of $u$ corresponding to the opening and closing of the ion channels, $\tau_{\mathrm{o} 1}=250.03, \tau_{\mathrm{o} 2}=16.632, \tau_{\mathrm{so} 2}=6.5537$, $k_{\mathrm{So}}=2.9748, \tau_{v 1}^{-}=16.3, \tau_{v 2}^{-}=1150, \tau_{v}^{+}=1.7026$, $\tau_{w 1}^{-}=79.963, \tau_{w 2}^{-}=28.136, \tau_{w}^{+}=213.55, \tau_{w \infty}=$ $0.2233, \tau_{\mathrm{s} 1}=9.876$, and $\tau_{\mathrm{s} 2}=4.2036$ are characteristic time for the opening $(+)$ and closing $(-)$ of the ionic channels (all in units of ms), $w_{\infty}^{*}=0.902, k_{s}=2.2268$, and $k_{w}^{-}=60.219$ are scaling parameters, $u_{s}=0.81568$ and $u_{w}^{-}=9.991 \times 10^{-3}$ are the respective shape parameters for the hyperbolic tangent in function $Q_{x}(u)$, and $\theta_{v}^{-}=0.1007$ and $\theta_{s}=\theta_{2}$ are additional threshold values for the opening and closing of the ionic channels. For modeling single-cell action potentials, we set $D=0$.

\section{Main parameters for fitting APs}

Recently, we showed that the four differential equations (1a) and (1b) can be effectively reduced to a system of two differential equations [5], that depends on the voltage $u$, together with exponentially decaying functions of the re- 
maining gating variables. This reduction shows that the three characteristic times $\tau_{\mathrm{fi}}, \tau_{\mathrm{si}}$ and $\tau_{\mathrm{sol}}$, in Eqs. (2a)-(2c), which fix the typical duration of the respective currents, are the most important parameters for the shape-modelling of APs. We take them as parameters for fitting a specific AP and keep all other parameters fixed. The values of the fixed parameters, given in the previous section, refer to the set determined for the electrically remodeled tissue due to atrial fibrillation $[2,6]$.

Through simulations of the BOCF model it is possible to extract the dependence of the APA and the $\mathrm{APD}_{n}$ with $n=20,40,50$ and 90 on the three characteristic times. Figure 2 shows these dependencies for a wide range of the parameter values, which we refer to as the "admissible range" in the following. Most of specific APs exhibit APA and $\mathrm{APD}_{n}$ values that are covered by the admissible ranges shown in Fig. 2. The $\mathrm{APD}_{90}$, however, becomes difficult to be correctly reproduced by our setting, if it exceeds $250 \mathrm{~ms}$ (vertical line in Fig. 1(a)). We refrained from modelling APs with $\mathrm{APD}_{90}$-values beyond this limit in the given setup. The limiting values are indicated in Fig. 1(b)-(f) by vertical lines. They correspond to the following ranges of the characteristic times: $\tau_{\mathrm{fi}} \in[0.002,0.21], \tau_{\mathrm{si}} \in[5,21.5]$ and $\tau_{\text {so } 1} \in[40,110]$ (in ms). Most of the APAs and APDs can be caught within these ranges.

It was shown [5] that the APA depends only very weakly on $\tau_{\mathrm{si}}$ and $\tau_{\mathrm{so} 1}$, and all APDs are almost independent of $\tau_{\mathrm{fi}}$. As shown in Fig. 2(a), the APA can be well described by a quadratic function of $\tau_{\mathrm{fi}}$ and the $\mathrm{APD}_{n}$ are well fitted by cubic polynomials of $\tau_{\mathrm{si}}$ and $\tau_{\mathrm{so1}}$ [5], as demonstrated in Figs. 2(b)-(e).

Because the quadratic fit of $\tau_{\mathrm{fi}}$ is monotonic in the admissible range of APA, it is uniquely determined by the APA. As for the other two times, we determine optimized

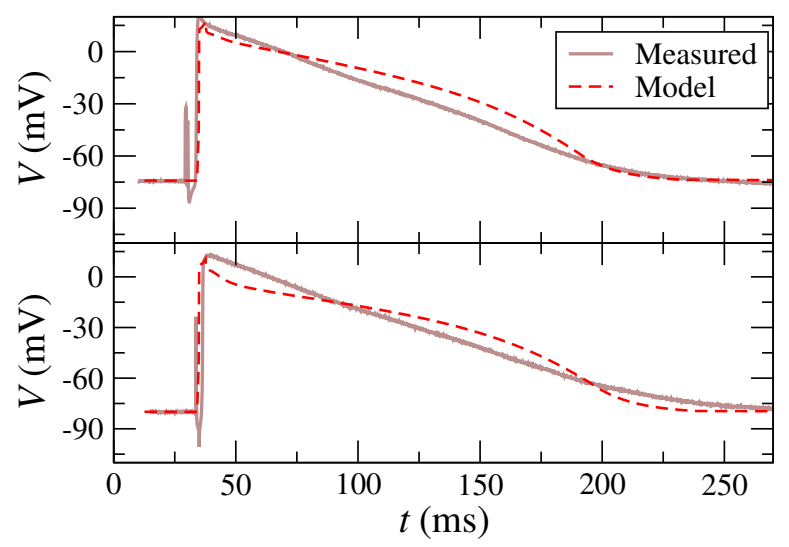

Figure 3. Two illustrative examples of the optimization procedure for fitting APs.
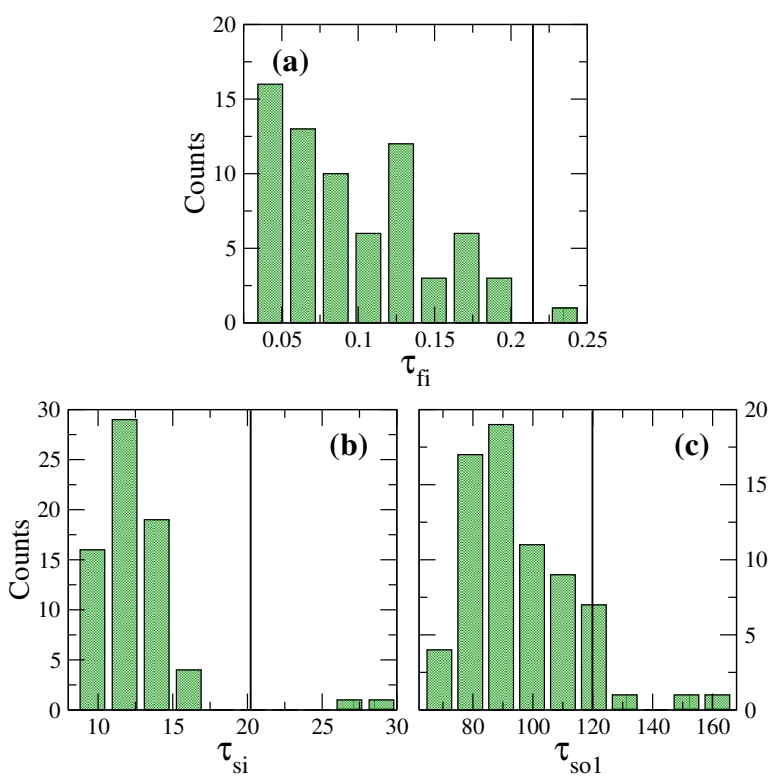

Figure 4. Distribution of the values of the three times obtained from the optimization procedure. Vertical lines indicate limiting values of the admissible range that can be properly modelled with the BOCF model (see Fig. 2).

values of $t a u_{s i}$ and $t a u_{s o 1}$ by minimizing the function

$$
\mathcal{F}\left(\tau_{\mathrm{si}}, \tau_{\mathrm{so} 1}\right)=\sum_{n}\left[\operatorname{APD}_{n}\left(\tau_{\mathrm{si}}, \tau_{\mathrm{so} 1}\right)-\mathcal{D}_{n}\right]^{2},
$$

where $\mathcal{D}_{n}$ are the measured values of the $\mathrm{APD}_{n}$. The minimization procedure is carried out numerically by employing the Levenberg-Marquadt algorithm. An analysis showed that the Hessian of the $\operatorname{APD}_{n}\left(\tau_{\mathrm{si}}, \tau_{\mathrm{so} 1}\right)$ is positive definite in the admissible parameter range. Thus, one expects to find a unique minimum of the function $\mathcal{F}\left(\tau_{\text {si }}, \tau_{\text {so1 } 1}\right)$.

\section{Modeling of patient-specific APs}

In this section we apply and evaluate the method by modelling the set of collected APs. Figure 3 shows two examples with the measured AP (solid black line) and the corresponding fit (dashed red line). By applying the method to the APs one obtains values for the three times, whose distribution is plotted in Fig. 4. The vertical lines indicate the respective boundary values (cf. Fig. 2). We discarded the APs with $\mathrm{APD}_{n}$ or APA values outside the admissible ranges, leaving 70 measured APs left for our modeling. Moreover, from this reduced set we took out six APs, having parameter values outside the ranges simulated with our framework (see vertical lines in Fig. 4).

For quantifying the deviations of the modelled APs from the measured ones, we compute their relative difference 


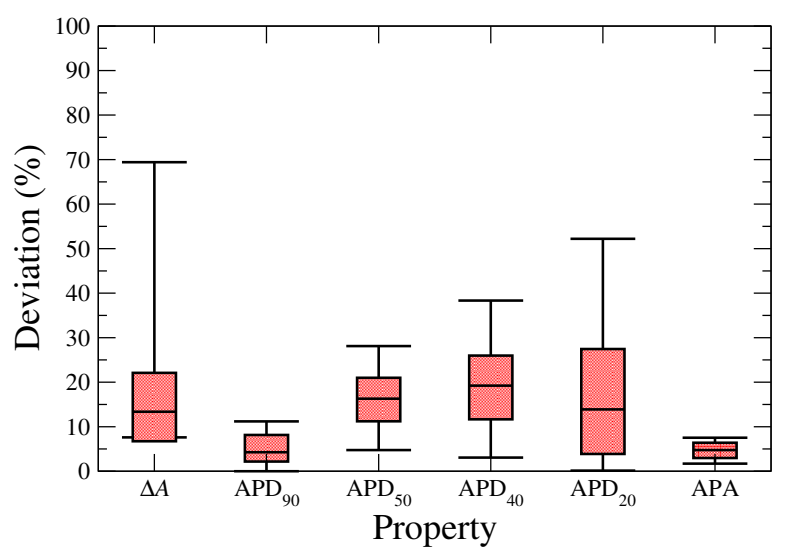

Figure 5. Box-plot of the distributions of deviations between the measured and the modelled AP parameters and of the $\Delta \mathcal{A}$ values quantifying the overall shape agreement.

based on the $L_{2}$-norm, namely

$$
\Delta \mathcal{A}=\frac{\left\|\mathcal{A}_{\mathrm{m}}(t)-\mathcal{A}_{\mathrm{e}}(t)\right\|_{L_{2}}}{\left\|\mathcal{A}_{\mathrm{e}}(t)\right\|_{L_{2}}}
$$

where $\mathcal{A}_{\mathrm{e}}(t)$ and $\mathcal{A}_{\mathrm{m}}(t)$ denote the measured and the modelled AP as a function of time, defined as $\|\mathcal{A}(t)\|_{L_{2}} \equiv$ $\left(\int_{t_{i}}^{t_{f}} \mathcal{A}^{2}(t) d t\right)^{1 / 2}$. The initial time $t_{i}$ and the final time $t_{f}$ are defined as the instants where $u\left(t_{i}\right)=u\left(t_{f}\right)=\theta_{0}$ with $\theta_{0}=0.015$ taken as a reference value. The time $t_{i}$ characterizes the rising flank of the $\mathrm{AP}$ and $t_{f}$.

Figure 5 shows that the typical deviations are of the order of $15 \%$. Moreover, we also compute the relative errors of the APA and $\mathrm{APD}_{n}$ defined as

$$
\Delta=\frac{\left|X_{\mathrm{m}}-X_{\mathrm{e}}\right|}{X_{\mathrm{e}}}
$$

with $X$ representing either the APA or one of the $\mathrm{APD}_{n}$. Deviations up to $20 \%$ are observed for the shortest $\mathrm{APD}_{n}$, namely for $n=20$ and 40 . The $\mathrm{APD}_{90}$ and the APA show mean deviations around $5 \%$.

\section{Conclusions}

We applied a method for modelling patient-specific APs based on the BOCF model, by adjusting three characteristic times, which are associated with the effective net sodium, calcium and potassium ionic currents introduced in this model. The method was applied to a set of 70 measured APs and showed deviations of the AP shape modeling of around $10 \%$. It demonstrates the possibilities of parameter adjustment of an atrial physiology model to reproduce AP shapes with a given amplitude, width and duration. While some large deviations are observed for short
APDs, the overall deviation of the modeled from the measured APs is comparable to that seen for AP modelling based on different detailed models (cf., e.g., Fig. 4 in [7]).

Showing a high flexibility for case-specific applications, our method can be useful for clinical purposes, because the three characteristic times retrieved by the underlying optimization procedure are directly connected to the iontype specific net currents. AP shapes showing pathological features will be reflected in the values of one (or more) times outside acceptable ranges, thus enabling to identify the class of membrane currents (sodium, calcium and/or potassium), where pathologies are likely to be present.

\section{Acknowledgements}

The authors thank Prof. U. Ravens for providing AP data and the Deutsche Forschungsgemeinschaft for financial support (Grant no. MA1636/8-1).

\section{References}

[1] A. Bueno-Orovio, E. M. Cherry, F. H. Fenton, "Minimal model for human ventricular action potentials in tissue", J. Theor. Bio. 253, 544-560 (2008).

[2] C. Lenk, F. M. Weber, M. Bauer, M. Einax, P. Maass, and G. Seemann, "Initiation of Atrial Fibrillation by Interaction of Pacemakers with Geometrical Constraints", J. Theor. Biol. 366, 13-23 (2015).

[3] Y. Richter, P. G. Lind, G. Seemann, P. Maass, "Anatomical and spiral wave reentry in a simplified model for atrial electrophysiology", J. Theor. Biol. 417, 100-107 (2017).

[4] M. Courtemanche, R.J. Ramirez, S. Nattel, "Ionic mechanisms underlying human atrial action potential properties: insights from a mathematical model”, Am. J. Phys. 275, H301-21 (1998).

[5] Y. Richter, P.G. Lind, P. Maass, "Optimized adjustment of a reaction-diffusion model to case-specific atrial physiology", submitted, 2017.

[6] G. Seemann, P. C. Bustamante, S. Ponto, M. Wilhelms, E. P. Scholz, O. Dössel, "Atrial Fibrillation-based Electrical Remodeling in a Computer Model of the Human Atrium", Comput. Cardiol. 37, 417-420 (2010).

[7] M. Wilhelms, H. Hettmann, M. M. Maleckar, J. T. Koivumäki, O. Dössel, G. Seemann, "Benchmarking electrophysiological models of human atrial myocytes", Front. Physiol. 3, 487 (2013).

Address for correspondence:

Name: Pedro G. Lind

Full postal address: Physics Department, University of Osnabrück, Barbarastrasse 7, 32/267, 49076 Osnabrück, Germany E-mail address: pedro.g.lind@gmail.com 